\title{
SQUARE PRINCIPLES WITH TAIL-END AGREEMENT
}

\author{
WILLIAM CHEN AND ITAY NEEMAN
}

\begin{abstract}
This paper investigates the principles $\square_{\lambda, \delta}^{\text {ta }}$, weakenings of $\square_{\lambda}$ which allow $\delta$ many clubs at each level but require them to agree on a tail-end. First, we prove that $\square_{\lambda,<\omega}^{\text {ta }}$ implies $\square_{\lambda}$. Then, by forcing from a model with a measurable cardinal, we show that $\square_{\lambda, 2}$ does not imply $\square_{\lambda, \delta}^{\text {ta }}$ for regular $\lambda$, and $\square_{\delta^{+}, \delta}^{\text {ta }}$ does not imply $\square_{\delta^{+},<\delta}$. With a supercompact cardinal the former result can be extended to singular $\lambda$, and the latter can be improved to show that $\square_{\lambda, \delta}^{\text {ta }}$ does not imply $\square_{\lambda,<\delta}$ for $\delta<\lambda$.
\end{abstract}

\section{INTRODUCTION}

Recently, Neeman [5] introduced the principles $\square_{\lambda, \delta}^{\text {ta }}$ and $\square_{\lambda,<\delta}^{\text {ta }}$, versions of Schimmerling's principles $\square_{\lambda, \delta}$ and $\square_{\lambda,<\delta}$ (see [6]) that require the clubs at each level of the sequence to agree on a tail-end. More precisely, for cardinals $\delta$ and $\lambda$, define a $\square_{\lambda, \delta}^{\text {ta }}$ sequence to be a sequence $\overrightarrow{\mathcal{C}}=\left\langle\mathcal{C}_{\alpha}: \alpha \in \operatorname{Lim}\left(\lambda^{+}\right)\right\rangle$such that for every $\alpha \in \operatorname{Lim}\left(\lambda^{+}\right)$,

(1) $\mathcal{C}_{\alpha}$ is a set of clubs of $\alpha, 1 \leq\left|\mathcal{C}_{\alpha}\right| \leq \delta$,

(2) for every $C \in \mathcal{C}_{\alpha}$, ot $(C)<\lambda$ if $\operatorname{cf}(\alpha)<\lambda$, and for every $\beta \in \operatorname{Lim}(C)$, $C \cap \beta \in \mathcal{C}_{\beta}$,

(3) for every $C, D \in \mathcal{C}_{\alpha}$ there exists $\beta<\alpha$ such that $C \backslash \beta=D \backslash \beta$.

The principle $\square_{\lambda, \delta}^{\text {ta }}$ asserts the existence of a $\square_{\lambda, \delta}^{\text {ta }}$ sequence. We also define $\square_{\lambda,<\delta}^{\text {ta }}$ asserting the existence of a sequence as above, except with $1 \leq\left|\mathcal{C}_{\alpha}\right|<\delta$. $\square_{\lambda, \delta}$ and $\square_{\lambda,<\delta}$ are defined in the same way, but without the tail-end agreement condition $(3)$.

Neeman observed that $\square_{\omega_{1}, \omega}^{\text {ta }}$ is strong enough to carry out a construction of Shelah-Stanley [7] of a $\omega_{2}$-Aronszajn tree which is not special (the construction originally used the principle $\square_{\omega_{1}}$ ). This is useful since $\square_{\omega_{1}, \omega}^{\text {ta }}$ follows from certain higher analogues of the proper forcing axiom, but these analogues do not imply $\square_{\omega_{1}}$. $\square^{\text {ta }}$ is strong enough to give some other consequences of $\square$. For example, it is not difficult to see that for any $\delta, \square_{\lambda, \delta}^{\text {ta }}$ implies that there is a nonreflecting stationary subset of $\lambda^{+}$, even though the weak square $\square_{\lambda, \lambda}$ does not.

Starting from a model with a Mahlo cardinal, Jensen [2] showed that $\square_{\lambda, \delta}$ does not imply $\square_{\lambda, \delta^{\prime}}$, where $\delta^{\prime}<\delta \leq \lambda$ and $\lambda$ is regular, and Cummings-ForemanMagidor [1] extended the result to singular $\lambda$ using a supercompact. Krueger and Schimmerling [3] showed $\square_{\lambda, \delta}$ does not imply $\square_{\lambda,<\delta}$ for $\delta \leq \lambda$, and also achieved separation results involving partial square principles.

2010 Mathematics Subject Classification. Primary 03E35; Secondary 03E05.

Key words and phrases. Forcing, combinatorial principles, square principles.

This material is based upon work supported by the National Science Foundation under Grant No. DMS-1101204. 
It is natural to ask where the square principles with tail-end agreement fit into this picture. It is easy to see that $\square_{\lambda, \delta}^{\text {ta }}$ implies $\square_{\lambda, \delta}$, and $\square_{\lambda}$ implies $\square_{\lambda, \delta}^{\text {ta }}$. In Section 2 , we will show that $\square_{\lambda,<\omega}^{\text {ta }}$ is actually equivalent to $\square_{\lambda}$. Section 3 proves $\square_{\lambda, \delta}^{\mathrm{ta}}$ is not implied by $\square_{\lambda, 2}$, and Section 4 proves that $\square_{\lambda, \delta}^{\mathrm{ta}}$ does not imply $\square_{\lambda,<\delta}$ for $\delta<\lambda$. In particular, the principle $\square_{\omega_{1}, \omega}^{\mathrm{ta}}$ considered in [5] is distinct from any of the square principles introduced in [6].

\section{2. $\square_{\lambda,<\omega}^{\text {ta }}$ IMPLIES $\square_{\lambda}$}

Suppose $\lambda$ is an uncountable cardinal.

Theorem 1. $\square_{\lambda,<\omega}^{\text {ta }}$ implies $\square_{\lambda}$.

Proof. Let $\overrightarrow{\mathcal{C}}$ be a $\square_{\lambda,<\omega}^{\text {ta }}$ sequence.

For each $\alpha \in \operatorname{Lim}\left(\lambda^{+}\right)$, set type $(\alpha)=\left|\mathcal{C}_{\alpha}\right|$. Define $g: \operatorname{Lim}\left(\lambda^{+}\right) \rightarrow \lambda^{+}$by $g(\alpha)=$ least $\beta$ such that $\left\{C \backslash \beta: C \in \mathcal{C}_{\alpha}\right\}$ is a singleton (so $g(\alpha)=0$ if type $(\alpha)=1$ ). Let $D_{\alpha}=C \backslash \beta$ for some (any) $C \in \mathcal{C}_{\alpha}$. Call $\alpha \in \lambda^{+} \operatorname{good}$ if $g\left\lceil\operatorname{Lim}\left(D_{\alpha}\right)\right.$ is bounded below $\alpha$, and let $G \subseteq \lambda^{+}$be the set of good points. Call $\alpha \in \lambda^{+}$bad if there is $k<\omega$ with $g \uparrow\left\{\beta \in \operatorname{Lim}\left(D_{\alpha}\right): \operatorname{type}(\beta)=k\right\}$ unbounded below $\alpha$, and let $B \subseteq \lambda^{+}$ be the set of bad points. Finally, call $\alpha \in \lambda^{+}$ugly if it is neither good nor bad, i.e., $g\left\lceil\operatorname{Lim}\left(D_{\alpha}\right)\right.$ is unbounded in $\alpha$ but $g\left\lceil\left\{\beta \in \operatorname{Lim}\left(D_{\alpha}\right): \operatorname{type}(\beta)=k\right\}\right.$ is bounded below $\alpha$ for all $k<\omega$. Let $U \subseteq \lambda^{+}$be the set of ugly points.

The first claim says that there are no ugly points of uncountable cofinality, allowing us to focus on good and bad points.

Claim 2.1. If $\alpha$ is ugly, then $\operatorname{cf}(\alpha)=\omega$.

Proof. For each $k<\omega$, let $\alpha_{k}=\sup \left\{g(\beta): \beta \in \operatorname{Lim}\left(D_{\alpha}\right)\right.$ and $\left.\operatorname{type}(\beta)=k\right\}$. Then since $\alpha$ is ugly, $\alpha_{k}<\alpha$ for every $k<\omega$ and $\sup \left\{\alpha_{k}: k<\omega\right\}=\alpha$.

The next claim will be used frequently in the arguments that follow.

Claim 2.2. Suppose $\alpha, \beta \in \operatorname{Lim}\left(\lambda^{+}\right)$and $\beta \in \operatorname{Lim}\left(D_{\alpha}\right)$. Then $\operatorname{type}(\alpha) \leq \operatorname{type}(\beta)$ and $g(\alpha) \leq g(\beta)$. If furthermore type $(\alpha)=\operatorname{type}(\beta)$, then $g(\alpha)=g(\beta)$.

Proof. Since $\beta \in \operatorname{Lim}\left(D_{\alpha}\right),\left|\left\{C \cap \beta: C \in \mathcal{C}_{\alpha}\right\}\right|=\operatorname{type}(\alpha)$. All of the clubs in this set must appear in $\mathcal{C}_{\beta}$, so type $(\alpha) \leq \operatorname{type}(\beta)$ and $g(\alpha) \leq g(\beta)$. In case $\operatorname{type}(\alpha)=\operatorname{type}(\beta), \mathcal{C}_{\beta}=\left\{C \cap \beta: C \in \mathcal{C}_{\alpha}\right\}$.

Now we begin the analysis of good and bad points.

Claim 2.3. If $\alpha$ is good, then all elements of $\operatorname{Lim}\left(D_{\alpha}\right)$ above a bound for $g \nmid \operatorname{Lim}\left(D_{\alpha}\right)$ are good. Furthermore, $g$ is eventually constant on $\operatorname{Lim}\left(D_{\alpha}\right)$.

Proof. By coherence, if $\beta \in \operatorname{Lim}\left(D_{\alpha}\right)$ is above a bound for $g \nmid \operatorname{Lim}\left(D_{\alpha}\right)$, then all elements of $\operatorname{Lim}\left(D_{\beta}\right)$ above that bound are also in $\operatorname{Lim}\left(D_{\alpha}\right)$, proving the first part of the claim. By Claim 2.2 and coherence, if $\beta<\gamma$ both belong to $\operatorname{Lim}\left(D_{\alpha}\right)$, and $\beta>g(\gamma)$, then $g(\beta) \geq g(\gamma)$. It follows that $g$ is non-increasing on $\operatorname{Lim}\left(D_{\alpha}\right)$ above a bound for $g\left\lceil\operatorname{Lim}\left(D_{\alpha}\right)\right.$, therefore it must be eventually constant.

If $\alpha$ is bad, define $k_{\alpha}<\omega$ to be the least $k$ such that $\left\{g(\beta): \beta \in \operatorname{Lim}\left(D_{\alpha}\right)\right.$ and $\operatorname{type}(\beta)=k\}$ is unbounded in $\alpha$. Note $k_{\alpha}>\operatorname{type}(\alpha)$ by Claim 2.2. Define an increasing continuous sequence $\left\langle\alpha_{\xi}\right\rangle \subset \operatorname{Lim}\left(D_{\alpha}\right)$ inductively. Set $\alpha_{0}$ to be the least $\gamma \in \operatorname{Lim}\left(D_{\alpha}\right)$ with type $(\gamma)=k_{\alpha}$, and $\alpha_{\xi+1}=$ the least $\gamma \in \operatorname{Lim}\left(D_{\alpha}\right)$ with $g(\gamma)>\alpha_{\xi}$ 
and type $(\gamma)=k_{\alpha}$. Note that $g\left(\alpha_{0}\right) \geq g(\alpha)$ by Claim 2.2. Let $E_{\alpha}$ be the range of this sequence.

The following claim can be thought of as a version of Claim 2.3 for bad points.

Claim 2.4. Suppose $\alpha$ is bad. Then $E_{\alpha}$ is closed unbounded in $\alpha$, and every point of $\operatorname{Lim}\left(E_{\alpha}\right)$ is bad and has type less than $k_{\alpha}$. Furthermore, $g$ is eventually constant on points of $\operatorname{Lim}\left(D_{\alpha}\right)$ of type $<k_{\alpha}$.

Proof. That $E_{\alpha}$ is closed unbounded in $\alpha$ follows immediately from the choice of $k_{\alpha}$ and the construction of $E_{\alpha}$. For the rest of the claim, observe that for every limit $\rho<\operatorname{ot}\left(E_{\alpha}\right)$, the sequence $\left\langle g\left(\alpha_{\xi}\right): \xi<\rho\right\rangle$ is unbounded in $\alpha_{\rho}$, and by coherence, a tail of $\left\langle\alpha_{\xi}: \xi<\rho\right\rangle$ is contained in $\operatorname{Lim}\left(D_{\alpha_{\rho}}\right)$. Therefore $\alpha_{\rho}$ is bad. Furthermore, the $\alpha_{\xi+1}$ are each by definition of type $k_{\alpha}$. By Claim 2.2, type $\left(\alpha_{\rho}\right) \leq k_{\alpha}$, and type $\left(\alpha_{\rho}\right) \neq k_{\alpha}$ otherwise $\left\langle g\left(\alpha_{\xi}\right): \xi<\rho\right\rangle$ could not be unbounded in $\alpha_{\rho}$.

The second part of the claim is proved similarly as Claim 2.3, working above a bound for $g$ restricted to points of $\operatorname{Lim}\left(D_{\alpha}\right)$ of type $<k_{\alpha}$ (which can be taken below $\alpha$ by minimality of $k_{\alpha}$ ).

If $\alpha$ is bad, then every $\beta \in \operatorname{Lim}\left(E_{\alpha}\right)$ is bad, so $k_{\beta}$ is defined. The next claim shows that above a certain bound, $k_{\beta}=k_{\alpha}$, and gives a weak coherence property between $E_{\alpha}$ and $E_{\beta}$ which will be useful later in our construction.

Claim 2.5. Suppose $\alpha$ is bad, and let $\alpha^{\prime}<\alpha$ be such that $g$ is constant on points of $\operatorname{Lim}\left(D_{\alpha}\right)$ of type $<k_{\alpha}$ which are greater than $\alpha^{\prime}$. Then for all $\beta \in \operatorname{Lim}\left(E_{\alpha}\right) \backslash \alpha^{\prime}$ we have $k_{\beta}=k_{\alpha}$ and $E_{\alpha} \cap(g(\beta), \beta)=E_{\beta}$.

Proof. Let $\beta \in \operatorname{Lim}\left(E_{\alpha}\right) \backslash \alpha^{\prime}$, say $\beta=\alpha_{\rho}$ for a limit ordinal $\rho$. By Claim 2.4, $\beta$ is bad. Moreover, $k_{\beta} \geq k_{\alpha}$ since $D_{\beta}$ and $D_{\alpha} \cap \beta$ are equal on a tail-end below $\beta$, and so $\left\{g(\gamma): \gamma \in \operatorname{Lim}\left(D_{\beta}\right)\right.$ and type $\left.(\gamma)<k_{\alpha}\right\}$ must be bounded below $\beta$ by the assumptions on $\alpha^{\prime}$. The reverse inequality $k_{\beta} \leq k_{\alpha}$ is witnessed by $\left\{\alpha_{\xi+1}: \xi<\rho\right\}$, which are all of type $k_{\alpha}$ by the construction.

By Claim 2.2, any $\gamma \in \operatorname{Lim}\left(D_{\beta}\right)$ has $g(\gamma) \geq g(\beta)$. By coherence, $D_{\beta}=D_{\alpha} \cap$ $[g(\beta), \beta)$. It follows that $\beta_{0}$ is the least $\gamma$ in $E_{\alpha}$ above $g(\beta)$ (where $\beta_{0}$ is the least member of $E_{\beta}$ ). Now $E_{\alpha}$ and $E_{\beta}$ are defined in the same way above $\beta_{0}$ by the coherence of $\overrightarrow{\mathcal{C}}$.

Extend the definition of $E_{\alpha}$ to all of $\operatorname{Lim}\left(\lambda^{+}\right)$by setting $E_{\alpha}=\operatorname{Lim}\left(D_{\alpha}\right)$ if $\alpha$ is good and ot $\left(D_{\alpha}\right)$ is a limit of limit ordinals, and $E_{\alpha}$ to be any sequence of order-type $\omega$ cofinal in $\alpha$ if $\alpha$ is ugly or ot $\left(D_{\alpha}\right)=\rho+\omega$ for some ordinal $\rho$.

We will define a function $h: \operatorname{Lim}\left(\lambda^{+}\right) \rightarrow \lambda^{+}$. If $\alpha$ is good and ot $\left(D_{\alpha}\right)$ is a limit of limits, set $h(\alpha)$ to be the least $\gamma \in \operatorname{Lim}\left(D_{\alpha}\right)$ such that $g$ is constant on $\operatorname{Lim}\left(D_{\alpha}\right) \backslash \gamma$. If $\alpha$ is bad, set $h(\alpha)$ to be the least $\gamma \in \operatorname{Lim}\left(D_{\alpha}\right)$ such that $g$ is constant on those points of $\left(\operatorname{Lim}\left(D_{\alpha}\right) \backslash \gamma\right)$ with type $<k_{\alpha}$. Otherwise, set $h(\alpha)=g(\alpha)$.

Finally, define $F_{\alpha}=E_{\alpha} \backslash h(\alpha)$ for each $\alpha \in \operatorname{Lim}\left(\lambda^{+}\right)$. We check that $\left\langle F_{\alpha}\right\rangle$ is a $\square_{\lambda}$ sequence.

Claim 2.6. For any $\alpha \in \operatorname{Lim}\left(\lambda^{+}\right), g(\alpha) \leq h(\alpha)<\alpha$, and for any $\beta \in \operatorname{Lim}\left(E_{\alpha} \backslash\right.$ $h(\alpha)$ ), we have $h(\beta)=h(\alpha)$.

Proof. The value of $h(\alpha)$ is either a point of $D_{\alpha}$ or just $g(\alpha)$, so $g(\alpha) \leq h(\alpha)$. The inequality $h(\alpha)<\alpha$ follows from Claim 2.3 or Claim 2.4, depending on the case.

Now we prove the second part of the claim. Suppose $\alpha$ is good and $\operatorname{ot}\left(D_{\alpha}\right)$ is a limit of limits. Then by definition of $h(\alpha)$ and the fact that $\beta \in \operatorname{Lim}\left(E_{\alpha}\right)=$ 
$\operatorname{Lim}\left(\operatorname{Lim}\left(D_{\alpha}\right)\right), \beta$ is also good and ot $\left(D_{\beta}\right)$ is a limit of limits. Above $h(\alpha), g$ is constant on $D_{\beta}$ with the eventual constant value of $g$ on $D_{\alpha}$. This value is also equal to $g(\beta)$, and by Claim 2.2, $g(\alpha) \leq g(\beta)$ so

$$
D_{\alpha} \cap(g(\beta), \beta)=D_{\beta} .
$$

The ordinal $h(\alpha)$ is defined to be an element of $\operatorname{Lim}\left(D_{\alpha}\right)$ with $g(h(\alpha))=g(\beta)$, so in particular $h(\alpha)>g(\beta)$. Together with $(2.1)$, this implies that $h(\beta)$ is computed using the same values as $h(\alpha)$, since $g(\alpha) \leq g(\beta)<h(\alpha)<\beta$. We conclude that $h(\beta)=h(\alpha)$.

The case where $\alpha$ is bad is similar: by Claim $2.5, \beta$ is bad with $k_{\beta}=k_{\alpha}$. Above $h(\alpha), g$ is constant on points of $D_{\beta}$ of type $<k_{\alpha}$ with the eventual constant value of $g$ on points of type $<k_{\alpha}$ in $D_{\alpha}$. This value is also equal to $g(\beta)$ since type $(\beta)<k_{\alpha}$ by Claim 2.4. By Claim 2.2, $D_{\alpha} \cap(g(\beta), \beta)=D_{\beta}$, so $h(\beta)$ is computed using the same values as $h(\alpha)$, and $h(\beta)=h(\alpha)$.

The claim is vacuously true for the remaining cases.

Suppose $\alpha \in \operatorname{Lim}\left(\lambda^{+}\right)$and $\beta \in \operatorname{Lim}\left(F_{\alpha}\right)$. If $\alpha$ is good, then $\beta$ is also good and using the fact that $g(\alpha), g(\beta) \leq h(\beta)$ we have

$$
F_{\beta}=\operatorname{Lim}\left(D_{\beta}\right) \backslash h(\beta)=\left(\operatorname{Lim}\left(D_{\alpha}\right) \cap \beta\right) \backslash h(\alpha)=F_{\alpha} \cap \beta .
$$

Similarly, if $\alpha$ is bad then $\beta$ is bad and we have

$$
F_{\beta}=E_{\beta} \backslash h(\beta)=\left(E_{\alpha} \cap \beta\right) \backslash h(\alpha)=F_{\alpha} \cap \beta .
$$

Here we used Claim 2.5 for the middle equality.

\section{3. $\square_{\lambda, 2}$ DOES NOT IMPLY $\square_{\lambda, \delta}^{\text {ta }}$}

Now we turn to separating $\square_{\lambda, \delta}^{\text {ta }}$ from the hierarchy of principles $\square_{\lambda, \delta^{\prime}}$ for various $\delta^{\prime}$. The methods we use, and the general structure of the proof, are similar to these used by [1] and [4] to separate square principles, and trace back to work of Jensen [2]. In this section we prove:

Theorem 2. Suppose $\lambda$ is an uncountable regular cardinal. If there is a measurable cardinal $\kappa>\lambda$, then there is a forcing extension preserving cardinals $\leq \lambda$ and $\geq \kappa$ in which $\square_{\lambda, 2}$ holds and $\square_{\lambda, \delta}^{\mathrm{ta}}$ fails for all $\delta$.

Proof. Let $\mathbb{P}$ the Levy collapse $\operatorname{Col}(\lambda,<\kappa)$. For this section, let $\mathbb{Q}$ be the poset in $V^{\mathbb{P}}$ forcing a $\square_{\lambda, 2}$-sequence using initial segments. More precisely, $\mathbb{Q}$ is the poset of all functions $q$ ordered by end-extension such that in $V^{\mathbb{P}}$,

(1) $\operatorname{dom}(q)=\operatorname{Lim}\left(\lambda^{+}\right) \cap(\alpha+1)$ for some limit ordinal $\alpha<\lambda^{+}$.

(2) For all $\beta \in \operatorname{dom}(q), q(\beta)$ is a set of closed unbounded subsets of $\beta$ of order type $\leq \lambda$, and $1 \leq|q(\beta)| \leq 2$.

(3) For all $\beta \in \operatorname{dom}(q)$, if $C \in q(\beta)$ and $\gamma \in \operatorname{Lim}(C)$, then $C \cap \gamma \in q(\gamma)$.

In $V^{\mathbb{P} * \mathbb{Q}}$ let $\overrightarrow{\mathcal{C}}$ be the $\square_{\lambda, 2}$ sequence added by $\mathbb{Q}$. Define $\mathbb{R}$ in $V^{\mathbb{P} * \mathbb{Q}}$ to be the poset of closed, bounded subsets $c \subseteq \kappa$ with the property that $c \cap \beta \in \mathcal{C}_{\beta}$ for any $\beta \in \operatorname{Lim}(c)$, ordered by end-extension. $\mathbb{R}$ adds a thread of $\overrightarrow{\mathcal{C}}$, i.e., a closed unbounded set $S \subseteq\left(\lambda^{+}\right)^{V^{\mathbb{P} * Q}}=\kappa$ such that $S \cap \beta \in \mathcal{C}_{\beta}$ for all $\beta \in \operatorname{Lim}(S)$.

Let $j: V \rightarrow M$ be an elementary embedding with $\operatorname{crit}(j)=\kappa$. We collect some useful facts about the various posets and their interactions with the embedding; proofs can be found in [4]. 
Fact 3.1. Let $G, H, I$ be generics for $\mathbb{P}, \mathbb{Q}, \mathbb{R}$, respectively.

- In $V[G], \mathbb{Q}$ is $\kappa$-distributive, and the set of flat conditions $\{(q, \check{r}) \in \mathbb{Q} *$ $\mathbb{R}: r \in V[G]$ and $\max (\operatorname{dom}(q))=\max (r)\}$ is dense and $\lambda$-closed. The condition $(q, \check{r})$ will be denoted as $(q, r)$ for simplicity.

- $j(\mathbb{P})=\operatorname{Col}(\lambda,<j(\kappa))$ and there is a complete embedding of $\mathbb{P} * \mathbb{Q} * \mathbb{R}$ into $j(\mathbb{P})$ with $\lambda$-closed quotient forcing,

- letting $J$ be generic for $j(\mathbb{P}) / \mathbb{P} * \mathbb{Q} * \mathbb{R}$, there is a $K$ generic for $j(\mathbb{Q})$ so that $j$ can be extended to an elementary embedding $j: V[G * H] \rightarrow M[G * H *$ $I * J * K]$ in the extension by $j(\mathbb{P} * \mathbb{Q})$.

In particular, all of the models we consider have the same $<\lambda$-sequences of ordinals.

We will show that $V[G * H]$ is a model satisfying the conclusion of the theorem. Clearly $\square_{\lambda, 2}$ holds in $V[G * H]$, so assume towards a contradiction that $\overrightarrow{\mathcal{D}}=\left\langle\mathcal{D}_{\alpha}\right.$ : $\alpha<\kappa\rangle$ is a $\square_{\lambda, \delta}^{\text {ta }}$ sequence in $V[G * H]$ for some $\delta$. Let $T \in j(\overrightarrow{\mathcal{D}})_{\kappa}$, so $T$ threads $\overrightarrow{\mathcal{D}}$ in $V[G * H * I * J * K]$. Since $j(\mathbb{Q})$ is $j(\kappa)$-distributive in $M[G * H * I * J], T$ must be a member of $M[G * H * I * J]$, and hence also $V[G * H * I * J]$.

Lemma 3.2. Suppose $V \subseteq W$ are models of set theory, $\lambda$ is an uncountable cardinal in $V$, and $V \vDash$ " $\overrightarrow{\mathcal{D}}$ is a $\square_{\lambda, \delta}^{\mathrm{ta}}$ sequence" for some $\delta$. Then forcing with a countably closed poset $\mathbb{S}$ over $W$ cannot add a new thread to $\overrightarrow{\mathcal{D}}$ (i.e., a thread not already in $W)$.

Proof. Assume towards a contradiction that $\dot{E}$ is an $\mathbb{S}$-name for a thread through $\overrightarrow{\mathcal{D}}$ which is forced to not be in $W$. Under this assumption, $\left(\lambda^{+}\right)^{V}$ has uncountable cofinality in $W$.

Claim 3.3. For any $\alpha<\left(\lambda^{+}\right)^{V}$, and $s_{0}, s_{1} \in \mathbb{S}$, there are $\beta>\alpha$ and $s_{0}^{\prime} \leq s_{0}, s_{1}^{\prime} \leq$ $s_{1}$ deciding " $\beta \in \dot{E}$ " differently.

Suppose that $s_{0}, s_{1}$, and $\alpha$ witness that this fails. Let $J_{0} \times J_{1}$ be generic for $\mathbb{S} \times \mathbb{S}$ over $W$ such that $\left(s_{0}, s_{1}\right) \in J_{0} \times J_{1}$. Then $\dot{E}\left[J_{0}\right]$ and $\dot{E}\left[J_{1}\right]$ have the same tail-end above $\alpha$, and since their proper initial segments belong to $W$ it follows that both belong to each of $W\left[J_{0}\right]$ and $W\left[J_{1}\right]$, and hence also $W$. This proves the claim.

Using the claim, we will recursively construct $s_{j}^{i} \in \mathbb{S}$ and ordinals $\alpha_{j}^{i}, \beta_{j}<\lambda^{+}$ for $i \in\{0,1\}$ and $j<\omega$ satisfying the following properties:

- $s_{j+1}^{i} \leq s_{j}^{i}$ and $\alpha_{j}^{0}<\alpha_{j}^{1}<\beta_{j}<\alpha_{j+1}^{0}$,

- $s_{j}^{0}$ and $s_{j}^{1}$ decide $\beta_{j} \in \dot{E}$ differently,

- $s_{j+1}^{i} \Vdash \alpha_{j+1}^{i} \in \dot{E}$.

By countable closure of $\mathbb{S}$, let $s^{0}$ be a lower bound for $\left\langle s_{j}^{0}: j\langle\omega\rangle, s^{1}\right.$ be a lower bound for $\left\langle s_{j}^{1}: j<\omega\right\rangle$, and $\beta^{*}=\sup \left\{\beta_{j}: j<\omega\right\}$. Note that $\beta^{*}<\left(\lambda^{+}\right)^{V}$, since $\left(\lambda^{+}\right)^{V}$ has uncountable cofinality in $W$. The values for $\dot{E}$ forced by $s^{0}$ and $s^{1}$ both have $\beta^{*}$ as a limit point, but for each $j<\omega$ they disagree on whether $\beta_{j} \in \dot{E}$. Since $\left\{\beta_{j}: j<\omega\right\}$ is cofinal in $\beta^{*}$ and $\dot{E}$ is forced to be a thread, this contradicts the tail-end agreement condition for $\overrightarrow{\mathcal{D}}$.

By Lemma 3.2, T must be a member of $V[G * H * I]$. For the remainder of the proof, work in $V[G]$ and let $\dot{T}$ be a $\mathbb{Q} * \mathbb{R}$-name for $T$. Note that a $\square_{\lambda, \delta}$ sequence in $V[G * H]$ cannot be threaded in $V[G * H]$ since all initial segments of the thread 
are initial segments of some $C_{\alpha}$ and thus have order-type $<\lambda$. Hence $T \notin V[G * H]$ and we get the following claim:

Claim 3.4. For any $q \in \mathbb{Q}, r_{0}, r_{1} \in \mathbb{R}, \alpha<\lambda^{+}$, there are $\beta>\alpha, q^{\prime} \leq q, r_{0}^{\prime} \leq$ $r_{0}, r_{1}^{\prime} \leq r_{1}$ such that $\left(q^{\prime}, r_{0}^{\prime}\right)$ and $\left(q^{\prime}, r_{1}^{\prime}\right)$ decide $" \beta \in \dot{T}$ " differently.

Proof. Suppose that $q, r_{0}, r_{1}$, and $\alpha$ witness that the claim fails. Modifying $H$ if necessary, we may assume $q \in H$. Working over $V[G * H]$, the argument proceeds as in the proof of Claim 3.3.

Let $(q, r) \in \mathbb{Q} * \mathbb{R}$ force that $\dot{T}$ threads $\overrightarrow{\mathcal{D}}$. Using Claim 3.4 and the fact that $\dot{T}$ is forced to be unbounded in $\lambda^{+}$, recursively construct flat conditions $\left(q_{j}, r_{j}^{i}\right) \in \mathbb{Q} * \mathbb{R}$ and ordinals $\alpha_{j}^{i}, \beta_{j}<\lambda^{+}$for $i \in\{0,1\}$ and $j<\omega$ satisfying the following properties:

- $\left(q_{j}, r_{j}^{i}\right) \leq(q, r)$,

- $\left(q_{j+1}, r_{j+1}^{i}\right) \leq\left(q_{j}, r_{j}^{i}\right)$, and $\alpha_{j}^{0}<\alpha_{j}^{1}<\beta_{j}<\alpha_{j+1}^{0}$,

- $\left(q_{j}, r_{j}^{0}\right)$ and $\left(q_{j}, r_{j}^{1}\right)$ decide $\beta_{j} \in \dot{T}$ differently,

- $\left(q_{j+1}, r_{j+1}^{i}\right) \Vdash \alpha_{j+1}^{i} \in \dot{T}$.

Now let $\gamma^{*}=\sup \left\{\max \operatorname{dom}\left(q_{j}\right): j<\omega\right\}$ and $\alpha^{*}=\sup \left\{\beta_{j}: j<\omega\right\}$. Define

$$
\begin{gathered}
\hat{r}^{i}=\bigcup\left\{r_{j}^{i}: j<\omega\right\} \cup\left\{\gamma^{*}\right\} \text { for } i \in\{0,1\}, \\
\hat{q}=\bigcup\left\{q_{j}^{i}: j<\omega\right\} \cup\left\{\left(\gamma^{*},\left\{\hat{r}^{0} \cap \gamma^{*}, \hat{r}^{1} \cap \gamma^{*}\right\}\right)\right\} .
\end{gathered}
$$

By the flatness we have maintained during the construction, we have for each $i \in\{0,1\}$ that $\gamma^{*}=\sup \left\{\max \operatorname{dom}\left(r_{j}^{i}\right): j<\omega\right\}$, so each $\left(\hat{q}, \hat{r}^{i}\right)$ is a condition in $\mathbb{Q} * \mathbb{R}$.

We can find $q^{*} \leq \hat{q}$ which decides the value of $\mathcal{D}_{\alpha^{*}}$, since no new subsets of $V[G]$ of size $<\lambda$ are added by $\mathbb{Q}$. For each $i \in\{0,1\},\left(q^{*}, \hat{r}^{i}\right) \Vdash$ " $\alpha^{*}$ is a limit point of $\dot{T}^{\text {" }}$ so $\left(q^{*}, \hat{r}^{i}\right) \Vdash \dot{T} \cap \alpha^{*} \in \mathcal{D}_{\alpha^{*}}$. But the values for $\dot{T}$ forced by $\left(q^{*}, \hat{r}^{0}\right)$ and $\left(q^{*}, \hat{r}^{1}\right)$ disagree on whether $\beta_{j} \in \dot{T}$, for each $j<\omega$. Since $\left\{\beta_{j}: j<\omega\right\}$ is cofinal in $\alpha^{*}$, this contradicts the tail-end agreement condition for $\overrightarrow{\mathcal{D}}$.

Starting with a supercompact cardinal instead of a measurable, we can get a version of Theorem 2 that applies to singular $\lambda$. This adapts the argument of Theorem 2 using ideas from Section 7 of [1].

Theorem 3. Suppose $\lambda$ is an infinite cardinal, $\mu$ is an uncountable regular cardinal $<\lambda$, and $\kappa$ is a supercompact cardinal with $\mu<\kappa \leq \lambda$. Then there is a forcing extension preserving cardinals in $\left[0, \mu^{+}\right] \cup\left[\kappa, \lambda^{+}\right]$in which $\square_{\lambda, 2}$ holds and $\square_{\lambda, \delta}^{\text {ta }}$ fails for all $\delta$.

Proof. We provide a rough sketch of the proof. Let $\mathbb{P}=\operatorname{Col}(\mu,<\kappa)$. Let $\mathbb{Q}$ be the poset defined in $V^{\mathbb{P}}$ forcing a $\square_{\lambda, 2}$ sequence using initial segments, and let $\overrightarrow{\mathcal{C}}$ be the $\square_{\lambda, 2}$ sequence added by $\mathbb{Q}$. Let $\mathbb{R}$ be the poset adding a thread through $\overrightarrow{\mathcal{C}}$ by closed initial segments of order-type $<\mu$.

If $G$ is generic for $\mathbb{P}$ and $H$ is generic for $\mathbb{Q}$, we claim that $V[G * H]$ is a model satisfying the conclusion of the theorem. Suppose for a contradiction that $\overrightarrow{\mathcal{D}}$ is a $\square_{\lambda, \delta}^{\text {ta }}$ sequence in $V[G * H]$ for some $\delta$. With $j: V \rightarrow M$ a $2^{\lambda}$-supercompactness embedding, it can be shown that there is some forcing extension of $V[G * H]$ by $j(\mathbb{P} * \mathbb{Q}) /(G * H)$ in which $j$ can be extended to $V[G * H]$. 
If $\overrightarrow{\mathcal{D}}$ is a $\square_{\lambda, \delta}^{\text {ta }}$ sequence, then define $\overrightarrow{\mathcal{D}}^{-\xi}$ by $\mathcal{D}_{\alpha}^{-\xi}=\mathcal{D}_{\alpha}$ if $\alpha \leq \xi$, and $\mathcal{D}_{\alpha}^{-\xi}=$ $\left\{C \backslash \xi: C \in \mathcal{D}_{\alpha}\right\}$ if $\alpha>\xi$. It is straightforward to check that $\overrightarrow{\mathcal{D}}^{-\xi}$ is still a $\square_{\lambda, \delta}^{\text {ta }}$ sequence.

Claim 3.5. If $\gamma=\sup j^{\text {" }} \lambda^{+}$and $A \in j(\overrightarrow{\mathcal{D}})_{\gamma}$, then there exists $\xi<\lambda^{+}$such that $T=\left\{\alpha \in \lambda^{+} \backslash(\xi+1): j(\alpha) \in \operatorname{Lim}(A)\right\}$ generates a thread through $\overrightarrow{\mathcal{D}}^{-\xi}$.

Since $j$ is continuous at points of countable cofinality, $j^{\text {" }} \lambda^{+}$is an $\omega$-club subset of $\gamma$ and hence $\operatorname{Lim}(A) \cap j^{\prime \prime} \lambda^{+}$is stationary in $\gamma$. The set $S=\left\{\alpha \in \lambda^{+}: j(\alpha) \in\right.$ $\operatorname{Lim}(A)\}$ is unbounded in $\lambda^{+}$since it is the pointwise $j$-preimage of $\operatorname{Lim}(A) \cap j^{\prime \prime} \lambda^{+}$. If $\alpha \in S$ then $A \cap j(\alpha) \in j(\overrightarrow{\mathcal{D}})_{j(\alpha)}=j\left(\mathcal{D}_{\alpha}\right)$. Let $\zeta(\alpha)$ be least so that there is $D_{\alpha} \in \mathcal{D}_{\alpha}$ such that $A \cap j(\alpha) \backslash \zeta(\alpha)=j\left(D_{\alpha}\right) \backslash \zeta(\alpha)$; by tail-end agreement for $j\left(\mathcal{D}_{\alpha}\right)$, $\zeta(\alpha)<j(\alpha)$. By Fodor's lemma, there is a stationary $B \subseteq \operatorname{Lim}(A) \cap j^{\prime \prime} \lambda^{+}$and $\zeta_{0}<\gamma$ so that $\zeta(\alpha)<\zeta_{0}$ if $j(\alpha) \in B$. Let $\xi<\lambda^{+}$be such that $j(\xi)>\zeta_{0}$.

If $\beta<\alpha$ are in $T=S \backslash(\xi+1)$, then $j\left(D_{\alpha}\right) \cap j(\beta) \backslash j(\xi)=A \cap j(\beta) \backslash j(\xi)=$ $j\left(D_{\beta}\right) \backslash j(\xi)$. By elementarity, $\beta \in \operatorname{Lim}\left(D_{\alpha}\right)$ and $D_{\beta} \backslash \xi=D_{\alpha} \cap \beta \backslash \xi$, so $\bigcup_{\alpha \in T} D_{\alpha} \backslash \xi$ threads $\overrightarrow{\mathcal{D}}^{-\xi}$. This proves the claim.

By the claim, replacing $\overrightarrow{\mathcal{D}}$ with $\overrightarrow{\mathcal{D}}^{-\xi}$, we may assume that $\left\{\alpha \in \lambda^{+} \backslash(\xi+1)\right.$ : $j(\alpha) \in \operatorname{Lim}(A)\}$ generates a thread through $\overrightarrow{\mathcal{D}}$. The poset $\mathbb{R}$ collapses $\lambda^{+}$to $\mu$ and can be absorbed into $j(\mathbb{P} * \mathbb{Q}) /(G * H)$. As in the proof of the previous theorem it can be shown that the thread through $\overrightarrow{\mathcal{D}}$ in the extension of $V[G * H]$ by $j(\mathbb{P} * \mathbb{Q}) /(G * H)$ must be added by $\mathbb{R}$, and that this leads to a contradiction.

Considering large $\delta$, all of the principles $\square_{\lambda, \delta}^{\text {ta }}$ with $\delta \geq \lambda^{+}$are equivalent. This can be easily seen by taking a $\square_{\lambda, \delta}^{\text {ta }}$ sequence $\overrightarrow{\mathcal{C}}$, and for each $\alpha \in \operatorname{Lim}(\lambda)$ fixing a particular $C_{\alpha} \in \mathcal{C}_{\alpha}$. Then define a $\square_{\lambda, \lambda^{+}}^{\text {ta }}$ sequence $\overrightarrow{\mathcal{D}}$ by $\mathcal{D}_{\beta}=\left\{C_{\alpha} \cap \beta: \beta \in\right.$ $\left.\operatorname{Lim}\left(C_{\alpha}\right)\right\}$. If $\lambda^{<\lambda}=\lambda$, then $\left|\mathcal{D}_{\alpha}\right| \leq \lambda$ for $\alpha<\lambda^{+}$of cofinality $<\lambda$ (and $\left|\mathcal{D}_{\alpha}\right|=1$ for $\alpha$ of cofinality $\lambda$ ), so $\square_{\lambda, \lambda^{+}}^{\text {ta }}$ and $\square_{\lambda, \lambda}^{\text {ta }}$ are also equivalent in this case.

This argument repeated with clubs not having to agree on a tail-end shows that $\square_{\lambda, \lambda^{+}}$is just outright true; however, Theorem 2 shows that with a measurable cardinal, even $\square_{\lambda, 2}$ does not imply $\square_{\lambda, \lambda^{+}}^{\mathrm{ta}}$.

\section{4. $\square_{\lambda, \delta}^{\text {ta }}$ DOES NOT IMPLY $\square_{\lambda,<\delta}$}

We will now show that $\square_{\lambda, \delta}^{\text {ta }}$ does not imply $\square_{\lambda,<\delta}$ for certain $\delta<\lambda$. Using a measurable cardinal, we will show:

Theorem 4. If $\delta$ is an infinite cardinal and there is a measurable cardinal $\kappa>\delta$, then there is a forcing extension preserving cardinals $\leq \delta^{+}$and cardinals $\geq \kappa$ in which $\square_{\delta^{+}, \delta}^{\mathrm{ta}}$ holds and $\square_{\delta^{+},<\delta}$ fails.

Strengthening the large cardinal hypothesis to a supercompact cardinal, we can obtain:

Theorem 5. Suppose $\delta<\lambda$ are infinite cardinals and there is a supercompact cardinal $\kappa$ with $\delta<\kappa \leq \lambda$. Then there is a forcing extension preserving cardinals in $\left[0, \delta^{+}\right] \cup\left[\kappa, \lambda^{+}\right]$in which $\square_{\lambda, \delta}^{\text {ta }}$ holds and $\square_{\lambda,<\delta}$ fails.

Theorem 5 does not apply when $\delta=\lambda$. If $\lambda$ is regular and not inaccessible, then Theorem 4 can be extended to this case. 
Theorem 6. Suppose $\lambda$ is an uncountable regular cardinal, $\lambda$ is not strongly inaccessible, and there is a measurable cardinal $\kappa>\lambda$. Then there is a forcing extension preserving cardinals $\leq \lambda$ and cardinals $\geq \kappa$ in which $\square_{\lambda, \lambda}^{\text {ta }}$ holds and $\square_{\lambda,<\lambda}$ fails.

Proof of Theorem 4. Let $\lambda=\delta^{+}$. We will force to add a $\square_{\lambda, \delta}^{\text {ta }}$ sequence with a certain extra property, and show that in the extension $\square_{\lambda,<\delta}$ fails. Let $\mathbb{P}=\operatorname{Col}(\lambda,<$ $\kappa)$ be the Levy collapse as in the last section, and $\mathbb{Q}$ be the poset defined in $V^{\mathbb{P}}$ of all functions $q$ ordered by end-extension such that

(i) $\operatorname{dom}(q)=\operatorname{Lim}\left(\lambda^{+}\right) \cap(\alpha+1)$ for some limit ordinal $\alpha<\lambda^{+}$.

(ii) For all $\beta \in \operatorname{dom}(q), q(\beta)$ is a set of closed unbounded subsets of $\beta$ of order type $\leq \lambda$, and $1 \leq|q(\beta)| \leq \delta$.

(iii) If $C \in q(\beta)$ and $\gamma \in \operatorname{Lim}(C)$, then $C \cap \gamma \in q(\gamma)$.

(iv) For every $C, D \in q(\beta)$ there exists $\beta<\alpha$ such that $C \backslash \beta=D \backslash \beta$.

(v) If $\operatorname{cf}(\beta) \leq \delta$, then for every $C \in q(\beta), \gamma \in \operatorname{Lim}(C)$, and $D \in q(\gamma)$,

$$
D \cup(C \backslash \gamma) \in q(\beta)
$$

In $V^{\mathbb{P} * \mathbb{Q}}$ let $\overrightarrow{\mathcal{C}}$ be the $\square_{\lambda, \delta}^{\text {ta }}$ sequence added by $\mathbb{Q}$. Define $\mathbb{R}$ to be the poset which adds a thread through $\overrightarrow{\mathcal{C}}$, i.e., the poset of closed bounded subsets $c \subseteq \kappa$ with the property that $c \cap \beta \in \mathcal{C}_{\beta}$ for any $\beta \in \operatorname{Lim}(c)$, ordered by end-extension.

Claim 4.1. Suppose $q$ satisfies (i)-(iv) in the definition of $\mathbb{Q}$ with $\operatorname{dom}(q)=$ $\operatorname{Lim}\left(\lambda^{+}\right) \cap \alpha+1$ for some $\alpha<\lambda^{+}$which is a limit of limit ordinals, $\operatorname{cf}(\alpha) \leq \delta$. Suppose further that for any limit ordinal $\beta<\alpha, q \uparrow(\beta+1) \in \mathbb{Q}$.

Define $q^{*}$ as the function on $\operatorname{dom}(q)$ with $q^{*} \uparrow \max (\operatorname{dom}(q))=q$ and

$$
q^{*}(\alpha)=q(\alpha) \cup\{D \cup(C \backslash \beta): C \in q(\alpha), \beta \in \operatorname{Lim}(C) \text { and } D \in q(\beta)\} .
$$

Then $q^{*} \in \mathbb{Q}$.

Proof. There are at most $\delta$ many $C \in q(\alpha)$ and $\delta$ many $\beta$ in each such $C$, so $\left|q^{*}(\alpha)\right| \leq \delta$. (It is important here that $\lambda=\delta^{+}$, for otherwise there could be more than $\delta$ many elements of $C$.) The only nontrivial requirements to check in the definition of $\mathbb{Q}$ are (iii) and (v) at $\alpha$.

To show (iii) at $\alpha$, suppose $E \in q^{*}(\alpha)$ and $\gamma \in \operatorname{Lim}(E)$. We check that $E \cap$ $\gamma \in q(\gamma)$. The less immediate case has $E=D \cup(C \backslash \beta)$ for some $C \in q(\alpha)$, $\beta \in \operatorname{Lim}(C)$, and $D \in q(\beta)$. If $\gamma \leq \beta$, then $E \cap \gamma=D \cap \gamma \in q(\gamma)$. If $\gamma>\beta$, then $E \cap \gamma=D \cup((C \cap \gamma) \backslash \beta)$. By (iii) applied at $\gamma, C \cap \gamma \in q(\gamma)$, so by (v) applied at $\gamma, E \cap \gamma=D \cup((C \cap \gamma) \backslash \beta) \in q(\gamma)$.

To show (v), suppose that $E \in q^{*}(\alpha), \gamma \in \operatorname{Lim}(E)$, and $F \in q(\gamma)$. We check that $F \cup(E \backslash \gamma) \in q^{*}(\alpha)$. Again, the less immediate case has $E=D \cup(C \backslash \beta)$ for some $C \in q(\alpha), \beta \in \operatorname{Lim}(C)$, and $D \in q(\beta)$. If $\gamma \geq \beta$, then $F \cup(E \backslash \gamma)=$ $F \cup(C \backslash \gamma) \in q^{*}(\alpha)$. If $\gamma<\beta$, then $\gamma \in \operatorname{Lim}(D)$, so by (v) applied at level $\beta$, we have $F^{\prime}:=F \cup(D \backslash \gamma) \in q(\beta)$. Therefore, $F \cup(E \backslash \gamma)=F^{\prime} \cup(C \backslash \gamma) \in q^{*}(\beta)$.

In the situation of the claim, we call $q^{*}$ the completion of $q$.

We have a version of Fact 3.1 for the new $\mathbb{Q}$ and $\mathbb{R}$. We will prove $\mathbb{Q}$ is $\kappa$ distributive by showing that it is $\lambda+1$-strategically closed (similarly to [1]). Recall that in our situation, $\kappa$ has been collapsed to be $\lambda^{+}$.

Lemma 4.2. The poset $\mathbb{Q}$ is $\lambda+1$-strategically closed, therefore $\kappa$-distributive. 
Proof. Players I and II play elements of $\mathbb{Q}$, with II playing at even stages, i.e., limit stages and even successor stages. We describe a winning strategy for player II. Let $q_{\xi}$ be the condition played at stage $\xi$ and $\beta_{\xi}$ be $\max \operatorname{dom}\left(q_{\xi}\right)$. At stage $\eta+2$, II plays $q_{\eta+2} \leq q_{\eta+1}$ with $\beta_{\eta+2}=\beta_{\eta+1}+\omega$ and $q_{\eta+2}\left(\beta_{\eta+2}\right)=\left\{\left\{\beta_{\eta+1}+n: 1 \leq n<\omega\right\}\right\}$.

If $\xi$ is limit, define $A_{\xi}=\left\{\beta_{\eta}: \eta<\xi\right.$ and $\eta$ even $\}$. II plays $q_{\xi}=\bigcup_{\eta<\xi} q_{\eta} \cup$ $\left\{\left(\beta_{\xi},\left\{A_{\xi}\right\}\right)\right\}$, with $\beta_{\xi}=\sup _{\eta<\xi} \beta_{\eta}$. This is closed and unbounded in $\xi$ by our construction so far. Furthermore, the construction ensures that for every $\gamma \in$ $\operatorname{Lim}\left(A_{\xi}\right), q_{\xi}(\gamma)$ is the singleton $\left\{A_{\xi} \cap \gamma\right\}$, so that coherence holds and condition (v) in the definition of $\mathbb{Q}$ is satisfied trivially at $\beta_{\xi}$.

The other parts of Fact 3.1 carry over to this situation as well.

Fact 4.3. Let $j: V \rightarrow M$ be an elementary embedding with $\operatorname{crit}(j)=\kappa$, and $G, H, I$ be generics for $\mathbb{P}, \mathbb{Q}, \mathbb{R}$, respectively.

- Working in $V^{\mathbb{P}}$, the set of flat conditions

$$
\{(q, \check{r}) \in \mathbb{Q} * \mathbb{R}: r \in V[G] \text { and } \max (\operatorname{dom}(q))=\max (r)\}
$$

is dense and $\lambda$-closed.

- There is a complete embedding of $\mathbb{P} * \mathbb{Q} * \mathbb{R}$ into $j(\mathbb{P})$ with $\lambda$-closed quotient forcing.

- Letting $J$ be generic for $j(\mathbb{P}) / \mathbb{P} * \mathbb{Q} * \mathbb{R}$, there is a $K$ generic for $j(\mathbb{Q})$ so that $j$ can be extended to an elementary embedding $j: V[G * H] \rightarrow$ $M[G * H * I * J * K]$ in the extension by $j(\mathbb{P} * \mathbb{Q})$.

Proof. We just prove the set of flat conditions is $\lambda$-closed, as this requires us to take a completion. Suppose $\left\langle\left(q_{\xi}, r_{\xi}\right): \xi<\eta\right\rangle$ is a decreasing sequence of flat conditions of $\mathbb{Q} * \mathbb{R}$, where $\eta<\lambda$. Letting $\alpha=\sup \left\{\max \operatorname{dom}\left(q_{\xi}\right): \xi<\eta\right\}, r=$ $\bigcup_{\xi} r_{\xi} \cup\{\alpha\}$, and $q$ be the completion of $\bigcup_{\xi} q_{\xi} \cup\{(\alpha, r \cap \alpha)\}$, we see that $(q, r)$ is a flat condition strengthening all the conditions from the sequence. The other parts of the claim are also proved just like the analogous facts in [4], taking completions where necessary.

As before, we will show that $V[G * H]$ is a model satisfying the conclusion of the theorem: $\square_{\lambda, \delta}^{\mathrm{ta}}$ holds in $V[G * H]$, so assume towards a contradiction that $\overrightarrow{\mathcal{D}}=\left\langle\mathcal{D}_{\alpha}: \alpha<\kappa\right\rangle$ is a $\square_{\lambda,<\delta}$ sequence in $V[G * H]$. Let $T \in j(\overrightarrow{\mathcal{D}})_{\kappa}$, so $T$ threads $\overrightarrow{\mathcal{D}}$ in $V[G * H * I * J * K]$.

The version of Lemma 3.2 we need here is essentially the same as Lemma 4.5 in [4], whose proof easily adapts to our statement.

Lemma 4.4. Suppose $V \subseteq W$ are models of set theory, $\lambda$ is an uncountable regular cardinal in $W$, and $\overrightarrow{\mathcal{D}}$ is $a \square_{\lambda,<\lambda}$ sequence in $V$. Then forcing with a $\lambda$-closed poset over $W$ cannot add a new thread to $\overrightarrow{\mathcal{D}}$.

By $j(\kappa)$-distributivity of $j(\mathbb{Q})$ and Lemma $4.4, T$ must be a member of $V[G *$ $H * I]$. Work in $V[G]$ and let $\dot{T}$ be a $\mathbb{Q} * \mathbb{R}$-name for $T$. Since $\overrightarrow{\mathcal{D}}$ is a $\square_{\lambda,<\delta}$ sequence in $V[G * H]$, it follows that $T \notin V[G * H]$, and therefore:

Claim 4.5. For any $q \in \mathbb{Q}, r \in \mathbb{R}$, there are $\alpha<\lambda^{+}, q^{\prime} \leq q, r_{0}^{\prime}, r_{1}^{\prime} \leq r$ such that $\left(q^{\prime}, r_{0}^{\prime}\right)$ and $\left(q^{\prime}, r_{1}^{\prime}\right)$ decide " $\alpha \in \dot{T}$ " differently.

Fix $f: \delta \rightarrow \delta$ such that $f(k) \leq k$ for each $k<\delta$, and for each $j<\delta$ there are unboundedly many $k<\delta$ with $f(k)=j$. We will recursively construct $\left\langle q_{j}: j \leq \delta\right\rangle$, $\left\langle r_{j}^{i}: i<j \leq \delta\right\rangle$, and $\left\langle\alpha_{j}: j<\delta\right\rangle$ such that for all $j \leq \delta$ : 
(1) $\left(q_{1}, r_{1}^{0}\right)$ forces that $\dot{T}$ is a thread of $\overrightarrow{\mathcal{C}}$.

(2) For all $i<j,\left(q_{j}, r_{j}^{i}\right) \in \mathbb{Q} * \mathbb{R}$ is flat and the order-type of $r_{j}^{i}$ is $\rho+1$ for some limit ordinal $\rho$. We will use the notation $\beta_{j}$ for $\max \left(\operatorname{dom}\left(q_{j}\right)\right)$.

(3) $\left\langle\alpha_{k}: k<\delta\right\rangle$ is a strictly increasing sequence of ordinals less than $\lambda^{+}$, and for each $i$ the sequence $\left\langle\left(q_{k}, r_{k}^{i}\right): i<k<\delta\right\rangle$ is decreasing in the $\mathbb{Q} * \mathbb{R}$ ordering,

(4) $\left(q_{j+1}, r_{j+1}^{f(j)}\right) \Vdash \alpha_{j} \in \dot{T}$.

(5) If $i, i^{\prime}<j$ are distinct, then $\left(q_{j}, r_{j}^{i}\right)$ and $\left(q_{j}, r_{j}^{i^{\prime}}\right)$ force distinct values for $\dot{T}$ below $\alpha_{j}$.

(6) If $i, i^{\prime}<j$, then $r_{j+1}^{i} \backslash \beta_{j}=r_{j+1}^{i^{\prime}} \backslash \beta_{j}$.

(7) If $j$ is limit, then $\beta_{j}=\sup \left\{\beta_{k}: k<j\right\}, r_{j}^{i}=\bigcup_{i<k<j} r_{k}^{i} \cup\left\{\beta_{j}\right\}$ for each $i<j$, and $q_{j}$ is the completion of $\bigcup_{k<j} q_{k} \cup\left\{\left(\beta_{j},\left\{r_{j}^{i} \cap \beta_{j}: i<j\right\}\right)\right\}$.

Assume that we are at stage $j+1$ of the construction, so that $\left(q_{j}, r_{j}^{i}\right)$ and $\alpha_{i}$ have been defined for all $i<j$. Using Claim 4.5, find $q_{j+1}^{\prime} \leq q_{j}, r_{j+1,0}, r_{j+1,1} \leq r_{j}^{0}$, and $\gamma<\lambda^{+}$such that $\left(q_{j+1}^{\prime}, r_{j+1,0}\right)$ and $\left(q_{j+1}^{\prime}, r_{j+1,1}\right)$ decide " $\gamma \in \dot{T}$ " differently. By extending further, we can take $\left(q_{j+1}^{\prime}, r_{j+1,0}\right)$ and $\left(q_{j+1}^{\prime}, r_{j+1,1}\right)$ to satisfy (2) above. Let $\beta_{j+1}^{\prime}=\max \operatorname{dom}\left(q_{j+1}^{\prime}\right)$.

We construct so that (4) holds. Since $r_{j+1,0} \in q_{j+1}^{\prime}\left(\beta_{j+1}^{\prime}\right)$, and $\beta_{j} \in \operatorname{Lim}\left(r_{j+1,0}\right)$ by (2), we can extend $\left(q_{j}, r_{j}^{f(j)}\right)$ to $\left(q_{j+1}^{\prime}, r_{j}^{f(j)} \cup\left(r_{j+1,0} \backslash \beta_{j}\right)\right)$ using (v) of the definition of $\mathbb{Q}$. Extend this to a condition which forces $\alpha_{j} \in \dot{T}$ for some $\alpha_{j}<\lambda^{+}$ with $\alpha_{j}>\gamma, \alpha_{j}>\alpha_{i}$ for every $i<j$. Extend further to $\left(q_{j+1}, r_{j+1}^{f(j)}\right)$ satisfying (2).

Set $r_{j+1}^{0}=r_{j+1,0} \cup\left(r_{j+1}^{f(j)} \backslash \beta_{j+1}^{\prime}\right)$ and $r_{j+1}^{j}=r_{j+1,1} \cup\left(r_{j+1}^{f(j)} \backslash \beta_{j+1}^{\prime}\right)$. For $0<i<j$, set $r_{j+1}^{i}=r_{j}^{i} \cup\left(r_{j+1}^{f(j)} \backslash \beta_{j}\right)$. By condition (v) from the definition of $\mathbb{Q}$, it follows that $\left(q_{j+1}, r_{j+1}^{i}\right) \in \mathbb{Q} * \mathbb{R}$ for all $i<j+1$.

Now suppose $j \leq \delta$ is limit. The construction is completely determined by (7). For any $i^{\prime}<i<j$ we have $r_{j}^{i} \backslash \beta_{i+1}=r_{j}^{i^{\prime}} \backslash \beta_{i+1}$, otherwise there is some $i<k<j$ where they disagree in $\left[\beta_{k}, \beta_{k+1}\right)$, contradicting (6). Therefore all of the $r_{j}^{i}$ agree on a tail-end and so $q_{j}$ defined by $(7)$ is really a member of $\mathbb{Q}$. It is straightforward to check inductively throughout that (1)-(7) above hold, so we have finished the construction.

Let $\alpha^{*}=\sup \left\{\alpha_{j}: j<\delta\right\}$. Find $q^{*} \leq q_{\delta}$ which decides the value of $\mathcal{D}_{\alpha^{*}}$. For all $i<\delta$,

$$
\left(q^{*}, r_{\delta}^{i}\right) \Vdash \alpha^{*} \text { is a limit point of } \dot{T}
$$

since $\left\{\alpha_{j}: f(j)=i\right\}$ is unbounded in $\alpha^{*}$ and $\left(q^{*}, r_{\delta}^{i}\right)$ forces such $\alpha_{j}$ into $\dot{T}$. This means $\left(q^{*}, r_{\delta}^{i}\right) \Vdash \dot{T} \cap \alpha^{*} \in \mathcal{D}_{\alpha^{*}}$. If $i \neq j$, then $\left(q^{*}, r_{\delta}^{i}\right)$ and $\left(q^{*}, r_{\delta}^{j}\right)$ force different values for $\dot{T} \cap \alpha^{*}$ by (5). This gives $\delta$ many distinct elements of $\mathcal{D}_{\alpha^{*}}$, a contradiction, concluding the proof of Theorem 4 .

This proof can be modified slightly to give Theorem 6 .

Proof of Theorem 6 . Let $\mu$ be the least cardinal such that $2^{\mu} \geq \lambda$. Since $\lambda$ is not strongly inaccessible, $\mu<\lambda$. Run the main construction in the proof of Theorem 4 for $\mu+1$ many steps, but with $i$ ranging over $2^{j}$ rather than $j$ at stage $j$. This involves modifying the successor step to extend each $r_{j}^{i}$, not just $r_{j}^{0}$, in two incompatible ways. At each limit stage $j<\mu$, there are fewer than $\lambda$ many $r_{j}^{i}$, so the 
construction can be continued. At stage $\mu$, take a subset of the $r_{\mu}^{i}$ of size $\lambda$ to form $q_{\mu}$. Then the argument is completed as in the proof of Theorem 4 .

The proof of Theorem 4 does not generalize immediately to the situation of Theorem 5, since closure of the set of flat conditions of $\mathbb{Q} * \mathbb{R}$ requires taking completions at limit levels, and therefore $\mathbb{Q} * \mathbb{R}$ (and hence also the quotient forcing $j(\mathbb{P}) / \mathbb{P} * \mathbb{Q} * \mathbb{R})$ is only $\delta^{+}$-closed. In the case where $\delta^{+}<\lambda$, this is insufficient to show that $T$ was not added by $j(\mathbb{P}) / \mathbb{P} * \mathbb{Q} * \mathbb{R}$. To overcome this, we will use a technique similar to the argument in Section 7 of [1] separating different $\square_{\lambda, \delta}$ for singular $\lambda$.

Proof of Theorem 5. Let $\mathbb{P}=\operatorname{Col}\left(\delta^{+},<\kappa\right)$. Let $\mathbb{Q}$ be the poset defined in $V^{\mathbb{P}}$ as in the proof of Theorem 4 , and let $\overrightarrow{\mathcal{C}}$ be the $\square_{\lambda, \delta}^{\text {ta }}$ sequence added by $\mathbb{Q}$. Let $\mathbb{R}$ be the poset adding a thread through $\overrightarrow{\mathcal{C}}$ by closed initial segments of order-type $<\delta^{+}$. It can be shown that the generic thread added by $\mathbb{R}$ has order-type $\delta^{+}$.

Again, we will build elements of $\mathbb{Q}$ by taking completions. The statement of Claim 4.1 holds in the new situation, but we must be more careful in the proof to avoid taking too many elements of $q^{*}(\alpha)$.

Claim 4.6. Suppose $q$ satisfies (i)-(iv) in the definition of $\mathbb{Q}$ with $\operatorname{dom}(q)=$ $\operatorname{Lim}\left(\lambda^{+}\right) \cap \alpha+1$ for some $\alpha<\lambda^{+}$which is a limit of limit ordinals, $\operatorname{cf}(\alpha) \leq \delta$. Suppose further that for any limit ordinal $\beta<\alpha, q \uparrow(\beta+1) \in \mathbb{Q}$.

Define $q^{*}$ as the function on $\operatorname{dom}(q)$ with $q^{*} \uparrow \max (\operatorname{dom}(q))=q$ and

$$
q^{*}(\alpha)=q(\alpha) \cup\{D \cup(C \backslash \beta): C \in q(\alpha), \beta \in \operatorname{Lim}(C) \text { and } D \in q(\beta)\} .
$$

Then $q^{*} \in \mathbb{Q}$.

Proof. Fix a particular $C_{0} \in q(\alpha)$. Assume that $\operatorname{Lim}\left(C_{0}\right)$ is unbounded in $\alpha$ (the other case is similar, and easier). Let $X$ be a subset of $\operatorname{Lim}\left(C_{0}\right)$ cofinal in $\alpha$ of ordertype $\operatorname{cf}(\alpha)$. Define $\tilde{q}^{*}(\alpha)=q(\alpha) \cup\{D \cup(C \backslash \beta): C \in q(\alpha), \beta \in \operatorname{Lim}(C) \cap X$ and $D \in$ $q(\beta)\}$. This has at most $\delta$ many elements.

We claim $q^{*}(\alpha) \subseteq \tilde{q}^{*}(\alpha)$. Suppose $C \in q(\alpha), \beta \in \operatorname{Lim}(C)$ and $D \in q(\beta)$. Then there is some $\gamma>\beta$ in $\operatorname{Lim}(C) \cap X$ since $X$ is unbounded in $\alpha$ and $C$ and $C_{0}$ agree on a tail-end. By condition (v) of the definition of $\mathbb{Q}$ and since $C \cap \gamma \in q(\gamma)$, $D^{\prime}=D \cup((C \cap \gamma) \backslash \beta) \in q(\gamma)$. Now $D \cup(C \backslash \beta)=D^{\prime} \cup(C \backslash \gamma) \in \tilde{q}^{*}(\alpha)$.

We get the basic facts about $\mathbb{P}, \mathbb{Q}, \mathbb{R}$ as before. In our new situation, let $j: V \rightarrow$ $M$ be a $2^{\lambda}$-supercompactness embedding.

Fact 4.7. Let $G, H, I$ be generics for $\mathbb{P}, \mathbb{Q}, \mathbb{R}$, respectively.

- In $V^{\mathbb{P}}$, the poset $\mathbb{Q}$ is $\lambda+1$-strategically closed, therefore $\kappa$-distributive.

- The set of flat conditions $\{(q, \check{r}) \in \mathbb{Q} * \mathbb{R}: r \in V[G]$ and $\max (\operatorname{dom}(q))=$ $\max (r)\}$ is dense and $\delta^{+}$-closed.

- There is a complete embedding of $\mathbb{P} * \mathbb{Q} * \mathbb{R}$ into $j(\mathbb{P})$ with $\delta^{+}$-closed quotient forcing.

- Letting $J$ be generic for $j(\mathbb{P}) / \mathbb{P} * \mathbb{Q} * \mathbb{R}$, there is a $K$ generic for $j(\mathbb{Q})$ so that $j$ can be extended to an elementary embedding $j: V[G * H] \rightarrow$ $M[G * H * I * J * K]$ in the extension by $j(\mathbb{P} * \mathbb{Q})$.

The first item is a parallel of Lemma 4.2. The second, which uses completions in an essential way and is therefore limited to $\delta^{+}$-closure, is a parallel of the first item 
of Fact 4.3. The remaining items are similar to facts found in [1], and the proofs there can be adapted to our situation in a straightforward way.

Assume towards a contradiction that $\overrightarrow{\mathcal{D}}$ is a $\square_{\lambda,<\delta}$ sequence in $V[G * H]$. Let $\gamma=\sup j^{\prime \prime} \lambda^{+}$and fix some $A \in j(\overrightarrow{\mathcal{D}})_{\gamma}$. Since $j(\mathbb{Q})$ is $j(\kappa)$-distributive, $A \in V[G *$ $H * I * J]$.

In this situation, we have an analogue of Claim 3.5 which gives a thread through $\overrightarrow{\mathcal{D}}$ in $V[G * H * I * J]$. The fact that $\delta<\kappa$ allows us to avoid the use of tail-end agreement for $\overrightarrow{\mathcal{D}}$ needed in the proof of Claim 3.5.

Claim 4.8. If $\gamma=\sup j^{\prime} \lambda^{+}$and $A \in j(\overrightarrow{\mathcal{D}})_{\gamma}$, then $S=\left\{\alpha \in \lambda^{+}: j(\alpha) \in \operatorname{Lim}(A)\right\}$ generates a thread $T$ through $\overrightarrow{\mathcal{D}}$.

Since $j$ is continuous at points of countable cofinality, $j^{\text {" }} \lambda^{+}$is an $\omega$-club subset of $\gamma$ and hence $\operatorname{Lim}(A) \cap j^{\prime \prime} \lambda^{+}$is unbounded in $\gamma$. Therefore, its pointwise $j$ preimage $S=\left\{\alpha<\lambda^{+}: j(\alpha) \in \operatorname{Lim}(A)\right\}$ is unbounded in $\lambda^{+}$. If $\alpha \in S$ then $A \cap j(\alpha) \in j(\overrightarrow{\mathcal{D}})_{j(\alpha)}=j\left(\mathcal{D}_{\alpha}\right)$. Since $\delta<\kappa, j\left(\mathcal{D}_{\alpha}\right)=j$ " $\mathcal{D}_{\alpha}$, so there is $D_{\alpha} \in \mathcal{D}_{\alpha}$ such that $A \cap j(\alpha)=j\left(D_{\alpha}\right)$. If $\beta<\alpha$ are in $S$, then $j\left(D_{\alpha}\right) \cap j(\beta)=A \cap j(\beta)=j\left(D_{\beta}\right)$. By elementarity, $\beta \in \operatorname{Lim}\left(D_{\alpha}\right)$ and $D_{\beta}=D_{\alpha} \cap \beta$, so $T=\bigcup_{\alpha \in S} D_{\alpha}$ threads $\overrightarrow{\mathcal{D}}$. This proves the claim.

We require a version of Lemma 4.4 which assumes less closure, and also applies to singular cardinals. The following is implicit in [1]:

Lemma 4.9. Let $\delta<\lambda$ be infinite cardinals. Suppose $V \subseteq W$ are models of set theory with the same cardinals $\leq \delta^{+}, W \vDash \operatorname{cf}\left(\left(\lambda^{+}\right)^{V}\right) \geq \overline{\delta^{+}}$, and $\overrightarrow{\mathcal{D}}$ is $a \square_{\lambda,<\delta}$ sequence in $V$. Then forcing with a $\delta^{+}$-closed poset over $W$ cannot add a new thread to $\overrightarrow{\mathcal{D}}$.

Since the thread added by $\mathbb{R}$ has order-type $\delta^{+}, V[G * H * I] \vDash \operatorname{cf}\left(\left(\lambda^{+}\right)^{V}\right)=\delta^{+}$. By Lemma 4.9, $T \in V[G * H * I]$. The rest of the proof proceeds in exactly the same way as the proof of Theorem 4 .

\section{REFERENCES}

[1] Cummings, J., Foreman, M., \& Magidor, M.: Squares, scales and stationary reflection. Journal of Mathematical Logic, 1(01), 35-98 (2001).

[2] Jensen, R. Some remarks on $\square$ below zero-pistol. Circulated notes.

[3] Krueger, J. \& Schimmerling, E.: Separating weak partial square principles. Annals of Pure and Applied Logic, 165(2), 609-619 (2014).

[4] Magidor, M., \& Lambie-Hanson, C.: On the strengths and weaknesses of weak squares. $A p$ palachian Set Theory: 2006-2012, 406, 301 (2012).

[5] Neeman, I. Two applications of finite side conditions at $\omega_{2}$.

[6] Schimmerling, E.: Combinatorial principles in the core model for one Woodin cardinal. Annals of Pure and Applied Logic, 74(2), 153-201 (1995).

[7] Shelah, S., \& Stanley, L.: Weakly compact cardinals and nonspecial Aronszajn trees. Proceedings of the American Mathematical Society, 104(3), 887-897 (1988).

Department of Mathematics, UCLA

E-mail address: chenwb@math.ucla.edu

Department of Mathematics, UCLA

E-mail address: ineeman@math.ucla.edu 